\title{
On Modeling and Analysis of MIMO Wireless Mesh Networks with Triangular Overlay Topology
}

\author{
Zhanmao Cao, ${ }^{1,2}$ Chase Q. Wu, ${ }^{2}$ Yuanping Zhang, ${ }^{3}$ Sajjan G. Shiva, ${ }^{2}$ and Yi Gu ${ }^{4}$ \\ ${ }^{1}$ Department of Computer Science, South China Normal University, Guangzhou, Guangdong 510631, China \\ ${ }^{2}$ Department of Computer Science, University of Memphis, Memphis, TN 38152, USA \\ ${ }^{3}$ School of Computer Science \& Education Software, Guangzhou University, Guangzhou, Guangdong 510006, China \\ ${ }^{4}$ Department of Computer Science, Middle Tennessee State University, Murfreesboro, TN 37132, USA
}

Correspondence should be addressed to Chase Q. Wu; chase.wu@memphis.edu

Received 27 September 2014; Revised 17 January 2015; Accepted 27 January 2015

Academic Editor: Hsuan-Ling Kao

Copyright (C) 2015 Zhanmao Cao et al. This is an open access article distributed under the Creative Commons Attribution License, which permits unrestricted use, distribution, and reproduction in any medium, provided the original work is properly cited.

\begin{abstract}
Multiple input multiple output (MIMO) wireless mesh networks (WMNs) aim to provide the last-mile broadband wireless access to the Internet. Along with the algorithmic development for WMNs, some fundamental mathematical problems also emerge in various aspects such as routing, scheduling, and channel assignment, all of which require an effective mathematical model and rigorous analysis of network properties. In this paper, we propose to employ Cartesian product of graphs (CPG) as a multichannel modeling approach and explore a set of unique properties of triangular WMNs. In each layer of CPG with a single channel, we design a node coordinate scheme that retains the symmetric property of triangular meshes and develop a function for the assignment of node identity numbers based on their coordinates. We also derive a necessary-sufficient condition for interference-free links and combinatorial formulas to determine the number of the shortest paths for channel realization in triangular WMNs.
\end{abstract}

\section{Introduction}

The WiMax group advocated the last-mile broadband services, IEEE 802.16 Standard [1], which defines broadband backbones as wireless mesh networks (WMNs). Such networks typically consist of two types of nodes, that is, base station (BS) and subscriber station (SS). BS is a wireless gateway connected to the Internet, while SS is a node that acts as a relay station. In multiple input multiple output (MIMO) WMNs, all nodes are equipped with multiple interfaces and support both multicast and mesh modes. Particularly, in a mesh mode, nodes can communicate with neighbors without the help of BS and the relay strategy provides an economical way to expand the mesh covering area. MIMO WMNs (in the rest of the paper, we use the term WMNs for conciseness) are well recognized as an efficient extension to the Internet backhaul [2].

WMNs possess some inherent characteristics that are different from ad hoc or wireless sensor networks. Since the nodes in WMNs are almost fixed and typically powered by electrical wires, the links or routing paths in WMNs generally last longer than those in mobile ad hoc networks. Also, every node in WMNs typically has nonzero traffic requests because it needs to route aggregated traffic from the terminal devices in its region for either upload or download. The topology of WMNs may be determined based on the predicted traffic requests or geographical environments. Since both BS and SS can be considered static, it is reasonable to view the mesh topology as a fixed graph.

The rapidly growing demand for ubiquitous Internet access requires an effective mathematical model for WMNs as it may simplify the tasks of routing, scheduling, and channel assignment. To achieve a maximum fair usage of multiple channels in WMNs, it is important to employ an efficient channel allocation scheme and an appropriate overlay graph topology for a given area [3]. In [4], the virtual topology is viewed as CPG to simplify the channel assignment problem through a graph. In addition, the CPG model also brings convenience for the analysis of routing and scheduling in WMNs. As nodes are static in WMNs, they can be identified 
geographically through their coordinates [5]. Therefore, the routing and scheduling problems can be analyzed using the node coordinates. Interference is another fundamental issue in either scheduling or routing, and the properties of interference under a given coordinate scheme, if defined properly and described rigorously, may bring benefits to resource utilization and interference avoidance.

In this work, we use CPG as a modeling approach and explore a set of unique properties of WMNs with a triangular topology. In each layer of CPG with a single channel, the network topology is a planar mesh. Our work makes several theoretical contributions to the analysis of WMN properties: (i) we design a coordinate scheme that retains the symmetric property of triangular meshes and develop a function to assign a unique identity number to a specific wireless node based on its coordinates. (ii) We derive a necessary-sufficient condition for interference-free links under the proposed node coordinate scheme. (iii) We derive combinatorial formulas in terms of the number of transceivers and channels to determine the number of the shortest paths for channel realization in triangular WMNs.

The rest of the paper is organized as follows. Section 2 surveys related work. Section 3 presents a channel-layered CPG model with emphasis on interference detection. In each layer of CPG, we propose a coordinate scheme, named parallel cluster coordinate, derive a necessary-sufficient condition for interference avoidance, and develop a function for node identity number assignment to support efficient WMN maintenance and administration. Section 4 derives formulas for determining the number of the shortest paths in WMNs.

\section{Related Work}

We conduct a brief survey of work directly related to mathematical models for WMNs.

In the past decade, most efforts in WMN topology were focused on interference and performance in planar meshes [6-9]. With geographical information from satellite or control channel communications, it is relatively easy to acquire a planar topology since the nodes in WMNs are almost static. For example, in IEEE Standard 802.21 for media-independent handover, Media Independent Information Service (MIIS) stores the geographical information of all access network operators available in a particular region [10].

Square and hexagonal meshes have been proposed to act as wireless broadband backbones [11]. However, they are less competitive than triangular meshes, as the latter outperforms the former and other random meshes in terms of various performance metrics such as coverage area, link quality, per-user fair rate, and node density [12]. Hong and Hua conducted a comparative evaluation of the throughput performance between square, hexagonal, and triangular meshes. Their experiments showed that triangular meshes achieve higher throughput than others in several cases, and their total throughput does not vary significantly in response to topology changes in large wireless networks with a constant density [13]. Therefore, we also adopt a triangular mesh topology in our model.
A unified network model based on super graph may further facilitate the analysis of various aspects of WMNs such as interference, scheduling, routing, and channel assignment. However, research efforts along this line are still quite limited. Several researchers considered some of these aspects simultaneously $[3,11,14]$, which motivates us to design a unified model for WMNs.

In a given network topology, a properly designed coordinate scheme may facilitate link interference detection and path finding. In hexagonal meshes, Chin et al. proposed a node coordinate scheme with three parallel line clusters [15], where a node is represented by a 3-tuple. In triangular meshes consisting of BS nodes with a node degree of six, Cao et al. proposed two BS-centered coordinate schemes [5] and explored interference and link groups in each of these schemes. Furthermore, in addition to coordinates, a router should also be assigned a unique identity number to support convenient simulation, administration, and maintenance. In $[4,5]$, Cao et al. also represented the coordinates of a node by a 3-tuple but did not tackle the identity number assignment problem.

The performance of WMNs is largely affected by link interference. Most research efforts on this subject have been made through generic methods or experimental studies, instead of conclusive results in the form of necessarysufficient conditions $[3,16,17]$. In our work, we attempt to design a suitable node coordinate scheme and then model the interference in WMNs as a specific checking list based on set theory.

Routing in WMNs is a 2-step procedure, that is, path finding followed by channel assignment. One basic approach to find an alternative interference-free path is to count the number of shortest paths and the number of all possible channel assignment schemes. A tree-like path finding scheme is proposed in [6] without any node coordinate. Cao and Xiao proposed path counting formulas for a source-destination pair in square grids [18], while the path counting problem in triangular meshes is still left unexplored. In our work, based on the proposed CPG model and coordinate scheme, we tackle this problem in triangular meshes with multiple channels and interfaces. Channel assignment is another important problem involving several network layers in WMNs, which is essentially an NP-complete edge coloring problem [14, 19].

\section{A Channel-Layered Graph Model}

As massive MIMO is on its way from theory to realistic deployment, one of the key problems is the interchannel cooperation, which calls for the development of sophisticated analytical channel models. Larsson et al. provide an overview of massive MIMO and motivate researchers to develop channel models capturing the essential channel behaviors despite their limitations [20]. For example, the Kronecker model, which is widely used to model channel correlation, is not an exact representation of reality but provides a useful model for certain types of analysis.

WMNs are conventionally modeled by a directed graph where a directed edge between two neighbor nodes represents a communication link over a specific channel. Since a node 


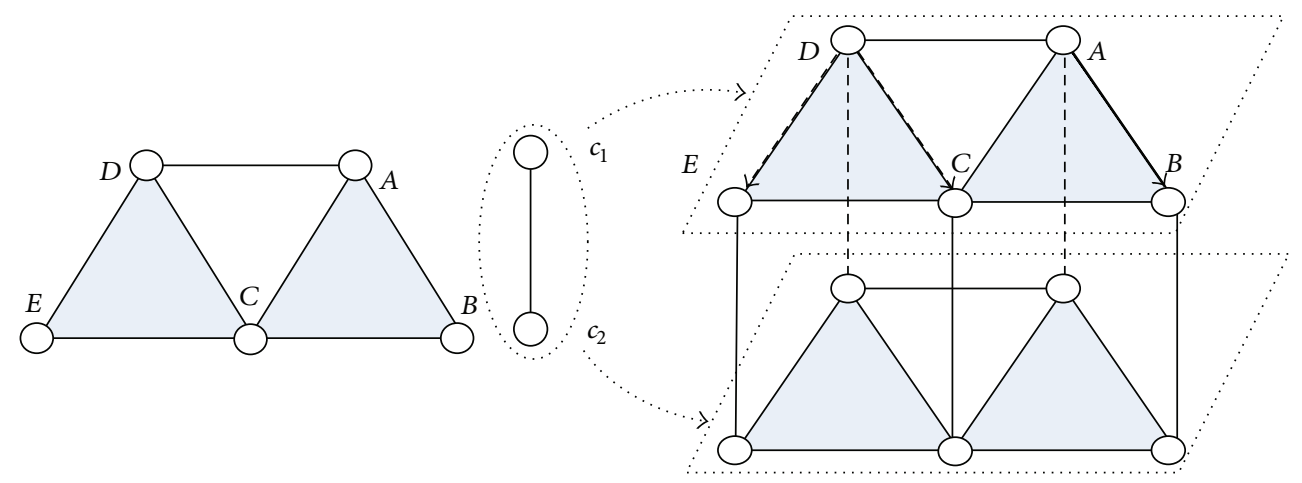

(a) Mesh and virtual nodes

(b) Layered mesh

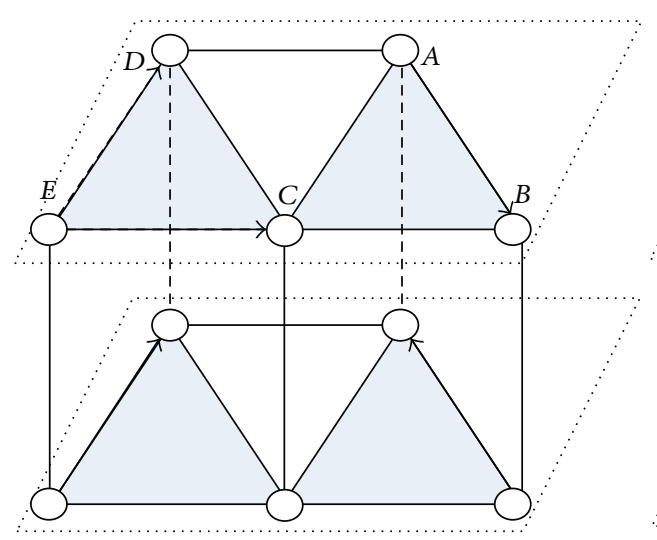

(c) Links in a channel

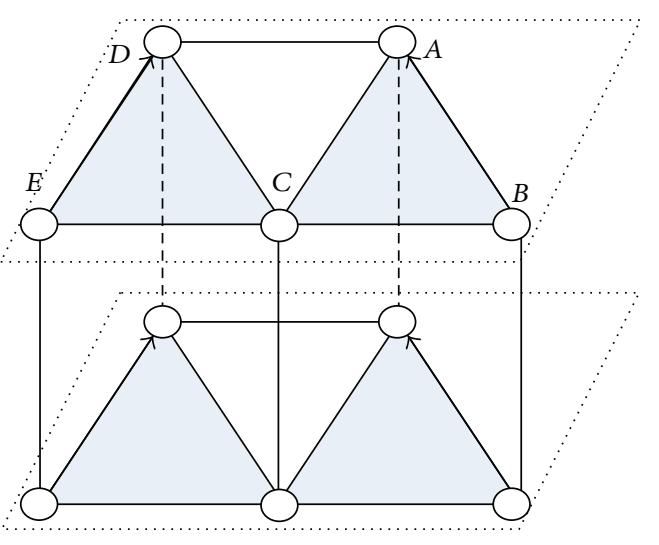

(d) Maximal links

FIGURE 1: The CPG-modeled virtual topology of a physical network with five physical nodes and two channels.

equipped with $\xi$ transceivers may have (at most) $\xi$ simultaneous links over $\xi$ orthogonal channels (assuming that more than $\xi$ channels are available), we can split a physical node into $\xi$ fully connected virtual nodes, each of which is equipped with a single transceiver. This way, we are able to represent the original WMN as $\xi$ identical layers of networks, each of which operates over a different channel.

3.1. Cartesian Product of Graphs. The topology-based modeling approach has been commonly used in wireless networks for various purposes, but often in a planar view $[8,12,14]$, and most of the discussions on scheduling, routing, and channel assignment are also based on a planar topology [6, $11,13,17]$. The recent development of MIMO WMNs calls for a suitable model to describe MIMO-specific properties and understand the cooperative activities across different interfaces over multiple channels [20]. The planar topology can be used to determine the internode interference [8] but is insufficient to provide a visual representation for analyzing the cooperation between links or channels. On the other hand, modeling MIMO WMNs as a super graph still remains largely unexploited except the work in [4]. In this paper, our goal is to develop an effective model to facilitate the analysis of MIMO channel cooperation.

We propose to employ the CPG to model WMNs by combining a triangular mesh of physical nodes and a graph of fully connected virtual nodes. Together with the coordinates of triangular overlay nodes, the CPG model provides a convenient way to analyze the properties of interference avoidance, channel assignment, and routing path counting. This model retains the independence between orthogonal channels while providing a general approach to analyzing link behaviors over multiple channels.

Cartesian Product of Graphs. Given two graphs $G$ and $H$, the Cartesian product $G \times H$ is a graph such that

(i) the graph $G \times H$ has a vertex set $V(G) \times V(H)$; that is, a vertex in $G \times H$ is denoted by a pair $\left(v, v^{\prime}\right), v \in V(G)$, and $v^{\prime} \in V(H)$;

(ii) any two nodes $\left(u, u^{\prime}\right)$ and $\left(v, v^{\prime}\right)$ and $u, v \in V(G)$ and $u^{\prime}, v^{\prime} \in V(H)$ are adjacent in $G \times H$, if and only if one of the following holds: (a) $u=v$ and $u^{\prime}$ is adjacent to $v^{\prime}$ in $H$, or (b) $u^{\prime}=v^{\prime}$ and $u$ is adjacent to $v$ in $G$.

For illustration, Figure 1(a) shows a mesh network of five physical nodes (left side) and a graph of two connected virtual nodes (right side) corresponding to a physical node equipped with two transceivers, each operating on a different channel $c_{1}$ or $c_{2}$. Figure $1(\mathrm{~b})$ shows a channel-layered virtual topology of the original mesh network modeled by CPG.

In this example, the CPG of two graphs in Figure 1(a) results in a two-layered graph in Figure 1(b). A solid directed 
edge in the top or bottom planar meshes in Figures 1(b), $1(\mathrm{c})$, and $1(\mathrm{~d})$ represents a communication link $l=(S, R)_{c}$, transmitting data from node $S$ to node $R$ over a channel $c$, while a dashed edge has a conflict with some active links.

The maximum possible number of concurrent active links on a given channel in a mesh is largely affected by the selection of the senders. As shown in Figure 1(b), when node $A$ in the top layer is sending data on channel $c_{1}$, the three neighbors of $A$, that is, nodes $B, C$, and $D$, in the same layer (over channel $c_{1}$ ) cannot send data. Furthermore, node $E$ can be selected as a sender, but neither of its neighbors, that is, nodes $C$ and $D$, can receive data from $E$ without interference, illustrated as the dashed directed edges in Figure 1(c). In this case, there is no other active link except $(A, B)_{c_{1}}$. However, if we choose the initial senders properly, as shown in Figure 1(d), there could exist two concurrent interference-free links on channel $c_{i}, i=1,2$, that is, $(B, A)_{c_{i}}$ and $(E, D)_{c_{i}}$.

Since all active links must be interference-free on the same channel at the same time, links are generally sparsely distributed in a planar mesh with respect to a certain channel. Note that more links mean better service to traffic requests. The CPG model allows us to consider concurrent paths in multiple layers over different channels through radio cooperation among channel layers for a given traffic task, hence providing more capacity and higher throughput compared to the situation with a single channel and radio. This is consistent with the experimental results presented by Draves et al. [16]. The proposed CPG model is aligned well with existing research in terms of interference relation, link activity, and network throughput, as well as routing and scheduling $[6,7,14,19,21]$ and enables us to conduct deeper theoretical analysis of WMNs.

One advantage that CPG brings is to simplify the expression of multichannel links. Since different layers (operating on different channels) are of the same topology, the scheduling strategy derived in one layer is readily applicable to another layer. For example, there exists a certain link distribution pattern among concurrent links. In CPG, it is obvious that such an interference-free link distribution pattern on one channel also exists on others.

3.2. Coordinate Scheme. A well-designed coordinate scheme may facilitate the analysis of WMNs. Since all channel layers are of the same topology, we only need to design the coordinate scheme for one layer or channel. The channel information can be added to the node coordinates to uniquely identify a specific layer.

Chin et al. proposed a coordinate scheme in hexagonal cellular networks where each node has a degree of three [15]. Inspired by their work, we propose a parallel cluster coordinate scheme in a local triangular mesh with one BS node and a number of SS nodes with a degree of six. This scheme can be readily extended to larger networks with multiple BSs by inserting the BS information to the node coordinates.

3.2.1. Parallel Cluster Coordinate Scheme. In a triangular mesh, we first define three clusters of parallel lines along three

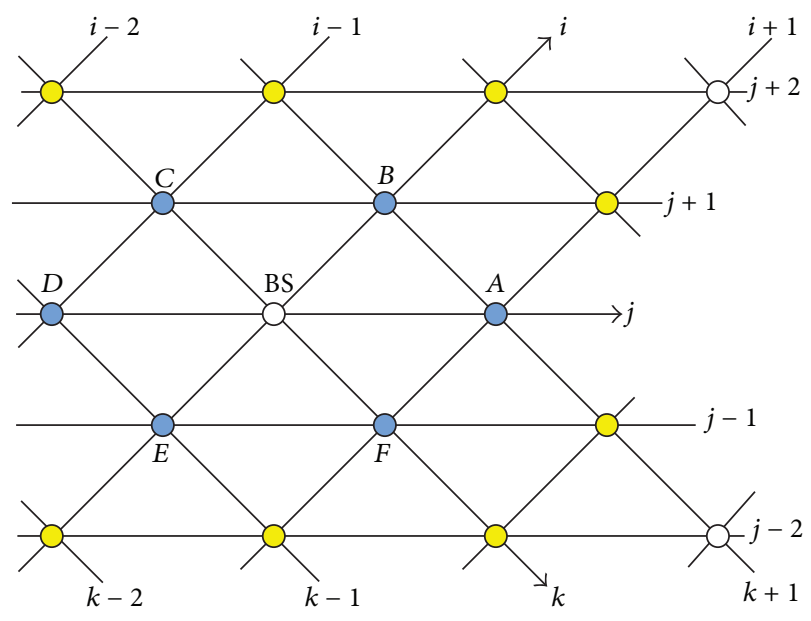

FIGURE 2: The parallel cluster coordinate scheme.

different directions, that is, north-east, east, and south-east. Since each node is a point intersected by three lines, each from one of the three clusters, we propose a parallel cluster coordinate scheme (PCCS), which uses a 3 -tuple $(i, j, k)$ to represent the coordinates of a node intersected by the $i$ th, $j$ th, and $k$ th line in the corresponding clusters [5]. As illustrated in Figure 2 , the BS node with the coordinates $(0,0,0)$ is located at the center, and the SS nodes $A, B, C, D, E$, and $F$, which are one hop away from the BS node, have the coordinates $A=(1,0,1), B=(0,1,1), C=(-1,-1,0), D=(-1,0,-1)$, $E=(0,-1,-1)$, and $F=(1,1,0)$.

Note that not every combination of three integers can represent a node in a triangular mesh because some lines defined by combinatorial 3-tuples do not intersect at a common point. We summarize such lines as follows:

$$
\begin{array}{r}
\Omega=\{(i-1, j-1, k),(i+1, j+1, k),(i, j-1, k+1), \\
(i, j+1, k-1),(i+1, j, k-1),(i-1, j, k+1)\} .
\end{array}
$$

Under the proposed PCCS scheme, for a given sender $(i, j, k)$, there are six possible receivers, which form its neighbor set $N_{b}(i, j, k)$ :

$$
\begin{aligned}
& N_{b}(i, j, k)=\{(i-1, j+1, k),(i+1, j-1, k), \\
&(i, j+1, k+1),(i, j-1, k-1), \\
&(i+1, j, k+1),(i-1, j, k-1)\} .
\end{aligned}
$$

PCCS retains the symmetric nature of a triangular mesh and facilitates the calculation of the distance between a pair of nodes.

3.2.2. Symmetric Property of PCCS. In PCCS, the coordinates of any two nodes that are symmetric with respect to the central BS node located at $(0,0,0)$ are negated. For example, the pairs of nodes $A$ and $D, B$ and $E$, and $C$ and $F$ in Figure 2 are symmetric, and their coordinates are negated from their counterparts.

The symmetric properties of CPG and Cayley graph have been well studied [22]. According to the vertex/edge 
transitive properties, a link group can be transited to generate another one in any layer.

Similarly, due to the symmetric property, we are able to transform a link group to another one through rotation. For example, link group of $((0,2,2),(0,1,1))_{c}$ and $((2,-2,0)$, $(1,-1,0))_{c}$ is interference-free. After a clockwise rotation of $\pi / 3$, a new group of interference-free links is of $((2,0,2)$, $(1,0,1))_{c}$ and $((0,-2,-2),(0,-1,-1))_{c}$, respectively. With another clockwise rotation of $\pi / 3$, the derived interferencefree links are $((2,-2,0),(1,-1,0))_{c}$ and $((-2,0,-2),(-1,0$, $-1))_{c}$, respectively. Note that the rotation operation is edge transitive, and it generates a new link group because of the symmetric property.

3.2.3. Distance between Two Nodes. As most traffic is upload/ download (to/from BS), we need to count the number of hops from a router node to BS in PCCS. We have the following properties.

Property 1. In triangular WMNs with PCCS, the minimum number $\hbar$ of hops from $A=(i, j, k)$ to $\mathrm{BS}=(0,0,0)$ is

$$
\hbar=\frac{|i|+|j|+|k|}{2}=\max \{|i|,|j|,|k|\}
$$

which is consistent with the one in [4].

Property 2. Suppose that BS is positioned at an arbitrary location $\left(i_{0}, j_{0}, k_{0}\right)$, instead of $(0,0,0)$. Node $(i, j, k)$ can be translated to $(i, j, k)-\left(i_{0}, j_{0}, k_{0}\right)$ by a translation function:

$$
\delta:(i, j, k) \longrightarrow\left((i, j, k)-\left(i_{0}, j_{0}, k_{0}\right)\right)
$$

In general, suppose that $\left(i_{0}, j_{0}, k_{0}\right)$ is the destination of a traffic path; we may virtually view $\left(i_{0}, j_{0}, k_{0}\right)$ as the BS node after applying the translation of (4). Then, the formula in (3) may facilitate further analysis.

Given two nodes $A=\left(i_{1}, j_{1}, k_{1}\right)$ and $B=\left(i_{2}, j_{2}, k_{2}\right)$ in PCCS, the distance between $A$ and $B$, denoted by $d(A, B)$, is the minimum number of hops between them, which is calculated as $d(A, B)=\left(\left|i_{1}-i_{2}\right|+\left|j_{1}-j_{2}\right|+\left|k_{1}-k_{2}\right|\right) / 2$.

3.2.4. Mapping to $2 D$ Points. In order to draw a planar mesh, we need to map 3-tuple coordinates $(i, j, k)$ to 2-dimensional (2D) points $(x, y)$. To do this, we first overlap a rectangular plane coordinate system to the PCCS triangular mesh. Let the $x$-axis overlap the axis $j=0$ while keeping the positive rightward direction. Meanwhile, the $y$-axis passes the BS node and is vertical to the line $j=0$ with a positive upward direction. Then, we can determine $(x, y)$ by projecting $(i, j, k)$ onto axes $x$ and $y$. The PCCS supports a function $f$ mapping $(i, j, k)$ to $(x, y)$ as follows.

Property 3. A one-to-one mapping function $f$ maps node coordinates $(i, j, k)$ in PCCS to $2 \mathrm{D}$ coordinates $(x, y)$ :

$$
(x, y)=f(i, j, k)=\left\{\begin{array}{l}
x=\frac{i+k}{2} \\
y=j .
\end{array}\right.
$$

For example, node $(0,1,1)$ is mapped to $(1 / 2,1)$, and $(-1,3,2)$ is mapped to $(1 / 2,3)$. If a node is on the $y$-axis, $i=$ $-k$ always holds. For example, $(-1,2,1)$ is mapped to $(0,2)$. If node is located on $k=0$ in PCCS, $x=i / 2$. For example, $(-2,2,0)$ is mapped to $(-1,2)$. This property facilitates the plotting of a triangular mesh in PCCS.

3.3. Interference-Free Conditions. To analyze the interference between links, we need to consider node interference relations, which are critical to scheduling links in WMNs. As interference is an inherent nature for radio media, the wireless communication performance may be severely degraded if radios operate without a proper scheduling scheme [17].

Minimizing interference has been extensively investigated in the literature $[9,17]$. Subramanian et al. discussed channel assignment in a multiradio situation [9]. Tan et al. designed algorithms to set up a skeleton of minimum interference for a single channel [17]. Scheduling links in a cooperative way will improve the energy efficiency and reduce collision. These discussions assume variable transmitting power or interface channel switching. However, the variation of transmitting power may lead to the variation of network topology, which may cause changes in the interference relation. Xu et al. use a sensing scheme to achieve power efficiency for convergence communication [23]. Their method can help set the initial power in an almost-static WMN topology while promising interference-free cognitive access with link status as busy or idle.

When sender $S$ is sending over channel $c_{0}$, neither can $S$ receive data over channel $c_{0}$, nor can its neighbors in its effective radio coverage send data. If a valid neighbor node $R$ receives data over channel $c_{0}$, we have a link $(S, R)_{\mathcal{C}_{0}}$. Furthermore, the neighbors of receiver $R$ cannot send data over channel $c_{0}$ at the same time. Therefore, to be link interference-free in multiradio multichannel environments using PCCS, we need to consider three classes of node interferences: sender-to-sender, receiver-to-receiver, and senderto-receiver.

3.3.1. Sender-to-Sender. Given a certain channel, any two senders must be at least two hops away in a triangular mesh to avoid mutual interference, which could help construct an interference-free candidate set for possible senders.

Definition 1. A router set $S_{C}$ is called a sender candidate set if every two nodes in $S_{C}$ are at least two hops away.

Given two nodes $S_{i}=(a, b, c)$ and $S_{j}=(d, e, f)$ in $S_{C}$, the distance between them must satisfy $d\left(S_{i}, S_{j}\right) \geq 2$.

For a given node $S_{j} \notin S_{C}$, we can add $S_{j}$ to $S_{C}$ only if it is at least two hops away to any node $S_{i}$ in $S_{C}$. We have the following necessary condition on interference-free links:

$$
d\left(S_{i}, S_{j}\right)=\frac{|a-d|+|b-e|+|c-f|}{2} \geq 2 .
$$

3.3.2. Receiver-to-Receiver. Two nodes $R_{i}$ and $R_{j}$ can each act as a receiver simultaneously if they have a distance larger than 1. Similar to the sender candidate set, we use $R_{C}$ to denote 
TABLE 1: Conditions for simultaneous links over one channel.

\begin{tabular}{lccc}
\hline \multicolumn{2}{c}{ Three neighbor pairs } & \multicolumn{2}{c}{ Link interference-free conditions } \\
Nodes & Nodes & Necessary & Sufficient \\
in $S_{C}$ & in $R_{C}$ & condition & condition \\
\hline$S_{i}, S_{j}$ & & $\forall i \neq j, d\left(S_{i}, S_{j}\right) \geq 2(1)$ \\
& $R_{i}, R_{j}$ & $\forall i \neq j, d\left(R_{i}, R_{j}\right) \geq 1(2)$ & $(1) \wedge(2) \wedge(3)$ \\
$S_{i}$ & $R_{j}$ & $\forall i \neq j, d\left(S_{i}, R_{j}\right)>1(3)$ & \\
\hline
\end{tabular}

a receiver candidate set, in which any two nodes $R_{i}=(l, m, n)$ and $R_{j}=(u, p, q)$ satisfy the following condition:

$$
d\left(R_{i}, R_{j}\right)=\frac{|l-u|+|m-p|+|n-q|}{2} \geq 1 .
$$

Note that both $S_{C}$ and $R_{C}$ are candidate sets, and the actual sender and receiver sets are a subset of $S_{C}$ and $R_{C}$, respectively. The relations between nodes largely depend on the previous selected links.

Property 4. If links $\left(S_{1}, R_{1}\right)_{c}$ and $\left(S_{2}, R_{2}\right)_{c}$ can be scheduled at the same time, then $d\left(S_{1}, S_{2}\right)$ must satisfy condition (6) and $d\left(R_{1}, R_{2}\right)$ must satisfy condition (7).

Note that Property 4 is only a necessary condition for interference-free links.

3.3.3. Sender-to-Receiver. Given a certain channel, a node cannot receive data if it is in the effective radio range of the sender of any other active link; otherwise, interference occurs. If two links $l_{i}$ and $l_{j}(i \neq j)$ coexist, the nodes involved in these two links must satisfy the following condition:

$$
d\left(S_{i}, R_{j}\right)=\frac{|a-u|+|b-p|+|c-q|}{2}>1, \quad i \neq j,
$$

where $S_{i}$ is the sender of link $l_{i}$ and $R_{j}$ is the receiver of link $l_{j}$.

For example, in Figure 1(d), since $A$ 's neighbor $D$ does not conflict with link $(B, A)_{\mathcal{C}_{1}}, D$ can be a receiver of another link $(E, D)_{\mathcal{C}_{1}}$.

We summarize three necessary conditions for link coexistence in the aforementioned three classes in Table 1.

We have the following theorem.

Theorem 2. Two links $l_{i}$ and $l_{j}$ can be simultaneously scheduled (coexist) on the same channel, if and only if they satisfy all of the three necessary conditions in Table 1.

Theorem 2 is based on the PCCS scheme and the set theory. As long as the PCCS node coordinates are given, we are able to determine the interference between links. These known conditions are helpful to find as many links as possible, while contributing to concurrent central scheduling.

3.4. Transformations of Link Groups. We attempt to find as many coexisting links as possible in a given local area. Since a link can be established only between a valid sender candidate and a valid receiver candidate, the actual (final)

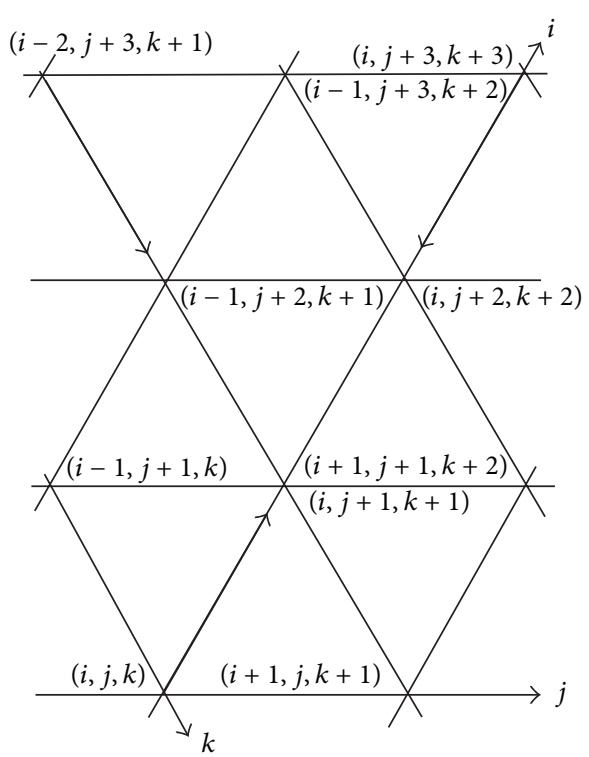

FIgure 3: Coexisting links in a local area.

scheduled link set is a subset of $S_{C} \times R_{C}$. Generally, coexisting links are sparsely distributed in the network. Based on a known group of coexisting links around one triangle, we wish to obtain a new group of coexisting links through certain transformations.

Starting from a link $l_{1}=\left(S_{1}, R_{1}\right)_{\mathcal{C}_{1}}$, where $S_{1}=(i, j, k)$ and $R_{1}=(i, j+1, k+1)$, we want to set up a dense link group in a local area. The set of $S_{1}$ 's neighbor nodes is $N_{b}\left(S_{1}\right)=\{v \mid$ $d(v,(i, j, k)) \leq 1, v \in V\}$. The set of $R_{1}$ 's neighbor nodes is $N_{b}\left(R_{1}\right)=\{v \mid d(v,(i, j+1, k+1)) \leq 1, \forall v \in V\}$. As any neighbor node of the sender or the receiver of an active link should remain silent if it does not receive data from the sender $S_{1}$, to avoid interference with $l_{1}$, nodes in $N_{b}(i, j, k) \cup N_{b}\left(R_{1}\right)$ cannot send data on $c_{1}$ when $l_{1}$ is active.

To expand the active link group containing $l_{1}=\left(S_{1}, R_{1}\right)_{c_{1}}$, two nodes $S_{2}=(i, j+3, k+3)$ and $R_{2}=(i, j+2, k+2)$, which are not in $N_{b}\left(S_{1}\right)$ and $N_{b}\left(R_{1}\right)$, may negotiate for a new link. If successful, $\left(S_{2}, R_{2}\right)_{\mathcal{c}_{1}}$ is added to the active link group. According to the conditions in Theorem 2, link $l_{2}=\left(S_{2}, R_{2}\right)_{\mathcal{c}_{1}}$ can coexist with $l_{1}$.

Any node in $N_{b}\left(R_{1}\right) \cup N_{b}\left(R_{2}\right)$ cannot be a new sender, except $S_{1}$ and $S_{2}$. However, it is completely different on the receiver side, where one node may be the receiver of a new link, even if it is in $N_{b}\left(R_{1}\right) \cap N_{b}\left(R_{2}\right)$. For example, in Figure 3, $S_{3}=(i-2, j+3, k+1)$ and $R_{3}=(i-1, j+2, k+1)$ form a new link $l_{3}=\left(S_{3}, R_{3}\right)_{\mathcal{C}_{1}}$, which can coexist with $l_{1}$ and $l_{2}$. Around the central triangle in Figure 3, we find three interference-free links coexisting over one channel.

Although the three nodes of the central triangle are involved in three active links as shown in Figure 3, neither of these three nodes can act as a sender while the other two are receiving data from their respective senders. To obtain the same number of new coexisting links, we need to keep the receivers unchanged, while considering a certain switching to the three senders of active links $l_{1}, l_{2}$, and $l_{3}$. 
By passing the sending token from the current sender to its neighbor node clockwise while keeping the original receiver, we are able to establish three possible new links:

$$
\begin{gathered}
l_{1}^{\prime}=((i-1, j+1, k),(i, j+1, k+1))_{c_{1}}, \\
l_{2}^{\prime}=((i+1, j+2, k+3),(i, j+2, k+2))_{c_{1}}, \\
l_{3}^{\prime}=((i-1, j+3, k+2),(i-1, j+2, k+1))_{c_{1}} .
\end{gathered}
$$

Since the sender $(i-1, j+3, k+2)$ of $l_{3}^{\prime}$ is one hop away from the receiver $(i, j+2, k+2)$ of $l_{2}^{\prime}$, which violates the third condition in Theorem $2, l_{2}^{\prime}$ and $l_{3}^{\prime}$ cannot coexist on channel $c_{1}$, which means that $l_{1}^{\prime}, l_{2}^{\prime}$, and $l_{3}^{\prime}$ cannot coexist. In other words, switching the senders of interference-free links may incur new interferences. Therefore, we must perform interference check after switching the sender of an active link.

Sender switching may generate a new dense link group in a local mesh. For example, we obtain a new link $l_{1}^{*}=((i+$ $1, j, k+1),(i, j+1, k+1))_{c_{1}}$, by switching the sender of link $l_{1}$ to its neighbor node anticlockwise. The three links $l_{1}^{*}, l_{2}$, and $l_{3}$ are interference-free. Similarly, $\operatorname{link} l_{3}^{*}=((i-2, j+2, k),(i-$ $1, j+2, k+1))_{c_{1}}$ is also interference-free with links $l_{1}$ and $l_{2}$.

Since triangular meshes possess symmetric properties, transformations such as rotation and translation can retain the interference-free features, which may save computing time in finding new link groups [24, 25].

3.5. Node Identity Number Assignment. The nodes in triangular meshes can be viewed not only as wireless router nodes, but also as resources or data sets. Assigning a unique identification (ID) number to each node brings several benefits. For example, such IDs can help to locate or identify nodes quickly for various administration or maintenance purposes.

Given the coordinates $(i, j, k)$ of a node in PCCS, there exists a general function $g$ that maps $(i, j, k)$ to a unique integer. For example, in Figure 2, we can map BS $=(0,0,0)$ to 0 , while $B=(1,0,-1)$ to 1 , and $C=(-1,1,0)$ to 2 .

Definition 3. In PCCS, $M$-circle in a triangular mesh is a set of nodes that have exact distance of $M$ hops to the node BS.

Suppose that $(i, j, k)$ is in $M$-circle, where $M=\max \{|i|$, $|j|,|k|\}$ according to (3). To construct a mapping function $g(i, j, k)$, we classify nodes according to their coordinates. For any node $A=(i, j, k)$, we consider the following seven cases in Figure 4, which is a logical route extracted from Figure 2. The first and special case $M=0, g(0,0,0)=0$. The node IDs in $M$-circle increase along an anticlockwise direction. These cases are applicable to any $M$-circle. The parallel solid line segments are corresponding to identity counting piecewise functions; for example, the 2nd case includes three parallel segments of different $M$-circle in Figure 4 , in which nodes on these segments get their ID number following the 2 nd one in (11). The dashed line means that the inner node is counted already, and the outer node is the new start of the next circle.

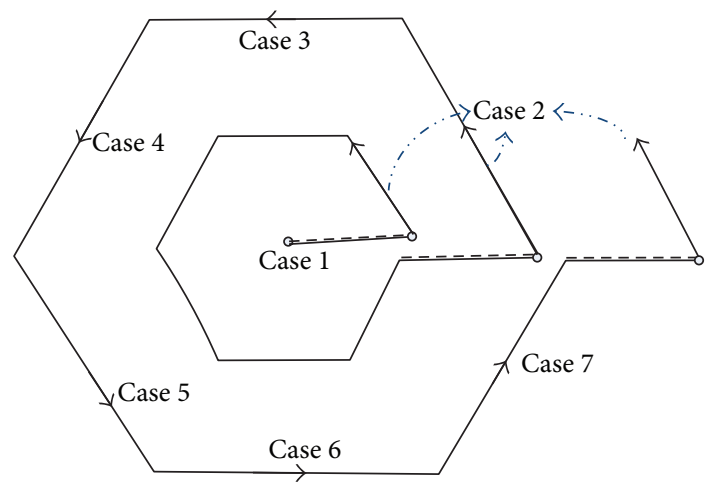

FIGURE 4: The cases for node identity number assignment.

To assign an identity to each node on $M$-circle, we need to know the total number $\kappa$ of nodes inside this circle:

$$
\kappa=1+6(1+2+\cdots+M-1)=1+3 M(M-1) .
$$

Let $\Delta=3 M(M-1)$; we have $\kappa=\Delta+1$. For example, there is one node inside circle $M=1$, there are $\Delta+1=7$ nodes inside circle $M=2$, and there are $\Delta+1=18+1=19$ nodes inside circle $M=3$.

The number of nodes on $M$-circle depends on the value of $M$. According to (11), for any $M$-circle, with the six solid line segments in the order as shown by arrows in Figure 4, we assign each node a unique integer on $M$-circle with an ID number in $\{\Delta+1, \Delta+2, \ldots, \Delta+j+1, \ldots, \Delta+6 M\}$. After overlapping Figure 4 on Figure 2, a node is assigned with its identity number in one of the seven cases in (11) corresponding to the line segment case in Figure 4:

$$
g(i, j, k)=\left\{\begin{array}{lll}
0 & M=0 & \text { 1st } \\
\Delta+j+1 & M \neq 0 \wedge M=k & \text { 2nd } \\
\Delta+M-i+1 & M \neq 0 \wedge M=j & \text { 3rd } \\
\Delta+2 M-k+1 & M \neq 0 \wedge M=-i & \text { 4th } \\
\Delta+3 M-j+1 & M \neq 0 \wedge M=-k & 5 \text { th } \\
\Delta+4 M+i+1 & M \neq 0 \wedge M=-j & \text { 6th } \\
\Delta+5 M+k+1 & M=i \wedge i \neq k, 0 & \text { 7th. }
\end{array}\right.
$$

For example, in the 6th case, node $(1,-4,-3)$ is assigned with an ID number of $3 \times 4 \times(4-1)+4 \times 4+1+1=54$.

Some nodes may satisfy two cases in (11) while getting the same ID. For example, node $(0,3,3)$, which is on the 3 -circle and the line segment in parallel with $k$-axis, is assigned with an ID number of $3 \times M \times(M-1)+j+1=3 \times 3 \times 2+3+1=$ 22 in the 2 nd case in (11). Meanwhile, node $(0,3,3)$, as it is on one line segment of Case 3 in Figure 4, can get its ID of $3 \times M \times(M-1)+M-i+1=3 \times 3 \times 2+3+1=22$ by following the 3 rd case in (11).

We shall provide more explanations for the 2nd and 7 th cases to facilitate a better understanding of (11). In the 2nd case where $M \neq 0 \wedge M=k$, the coordinate $k$ of node $(i, j, k)$ must be positive. Node $(i, j, k)$ on this $M$-circle $(M=k)$ segment should be assigned with an ID number in 
$\{\Delta+1, \Delta+2, \ldots, \Delta+j+1, \ldots, \Delta+M\}$. In this case, node $(i, j, k)$ is located above the lines $j=0$ and $k>0$, as shown in Case 2 in Figure 4 . Hence, with the increasing $j$, each node $(i, j, k)$ is assigned with an ID number $g(i, j, k)=\Delta+j+1$. Generally, on each $M$-circle $(M>0)$, from the 2 nd case to the 7 th case, each with $M$ nodes, the total number of nodes is $6 M$.

In the 7 th case, we need to avoid repeatedly counting the node on line $j=0$, as shown in Figure 2. The number of hops to the BS is $M=i$. The last node on this circle is on a line segment of the $i$-axis parallel cluster with $j=0$ according to Figure 4 . The condition $M=i \wedge i \neq k$ excludes the nodes already counted in the previous Case 2 in this circle, while $M=i \wedge i \neq 0$ prevents the 1st case from being reconsidered. Meanwhile, the condition $M \neq k$ ensures that we count nodes on the next circle following the 2 nd case again where $M=k$. In the 7 th case, a node can be assigned with an ID number in a finite set $\{\Delta+5 M+1, \ldots, \Delta+5 M+k+1, \ldots, \Delta+6 M\}$. With the increasing $k$, where $k=0,1, \ldots, M-1$, node $(i, j, k)$ on this segment is assigned with an ID number of $\Delta+5 M+k+1$.

Note that the mapping function $g(i, j, k)$ is a segmented linear function, which is invertible for any finite set.

\section{Path Counting}

Routing is one fundamental problem in WMNs. To develop a good routing scheme, one needs to know the number of alternative paths and the number of channel assignments for a given pair of source and destination. We discuss path finding and realization based on the proposed CPG model and coordinate scheme. The total number of shortest paths in grid meshes was discussed in [18]. In this section, we tackle the path counting problem in triangular meshes.

We use $d(S, D)$ to denote the distance between source $S=$ $\left(i_{1}, j_{1}, k_{1}\right)$ and destination $D=\left(i_{2}, j_{2}, k_{2}\right)$. Firstly, $d(S, D)=$ $\left(\left|i_{1}-i_{2}\right|+\left|j_{1}-j_{2}\right|+\left|k_{1}-k_{2}\right|\right) / 2$. In order to transmit data from $S$ to $D$, we need to select one path from $P(S, D)$, which is the set of all shortest paths from $S$ to $D$.

4.1. Path Alternatives. Every step along the shortest path from $S$ to $D$ is one hop forward in one of the directions $i, j$, and $k$. The two smaller numbers of $\left\{\left|i_{1}-i_{2}\right|,\left|j_{1}-j_{2}\right|,\left|k_{1}-k_{2}\right|\right\}$ indicate the lines of parallel clusters that form a grid mesh for path selection, as illustrated in the grid of dashed lines in Figure 5. We refer to the two corresponding directions from $S$ to $D$ in the grid mesh as the correct directions.

The correct directions ensure that the data is transmitted through one of the shortest path, where every hop selection makes one hop closer to the destination. Through the use of correct directions, we are able to reduce the problem of counting all shortest paths from $S$ to $D$ in triangular meshes to a problem in grid meshes.

We provide an example in Figure 5 to count the number of paths, where $\left|i_{1}-i_{2}\right|=2,\left|j_{1}-j_{2}\right|=3$, and $\left|k_{1}-k_{2}\right|=5$. The grid with dashed lines contains all the shortest paths from $S$ to $D$.

In a simple situation where one of $\left\{\left|i_{1}-i_{2}\right|,\left|j_{1}-j_{2}\right|, \mid k_{1}-\right.$ $\left.k_{2} \mid\right\}$ is 0 , the dashed grid degrades to a line. Therefore, there is only one shortest path available.

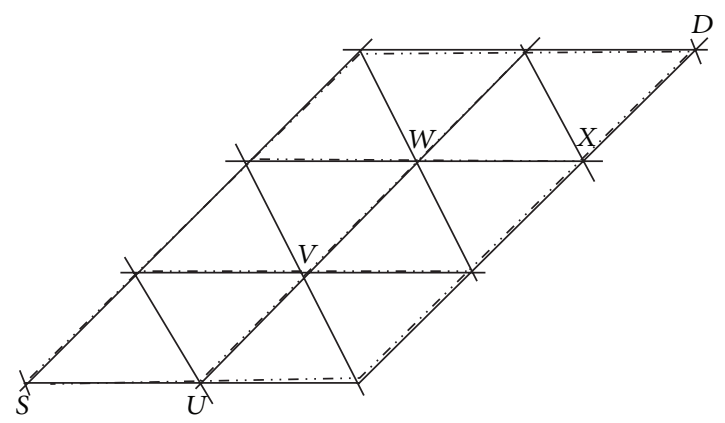

FIGURE 5: The shortest path alternatives in triangular meshes.

For convenience, let $\wp=\left\{\left|i_{1}-i_{2}\right|,\left|j_{1}-j_{2}\right|, \mid k_{1}-\right.$ $\left.k_{2} \mid\right\}$. The correct directions are consistent with $\min (\wp)$ (the minimum of three elements in $\wp$ ) and $\operatorname{mid}(\wp)$ (the middle of three elements in $\wp$ ). To select a shortest path, it is necessary to remove the direction corresponding to $\max (\wp)$ (the maximum of three elements in $\wp$ ); otherwise, it would lead to a longer path.

If the smallest in $\left\{\left|i_{1}-i_{2}\right|,\left|j_{1}-j_{2}\right|,\left|k_{1}-k_{2}\right|\right\}$ is 1 or 2 , in the corresponding direction, the sender and receiver must be on two neighbor parallel lines or two parallel lines with one line between them. We can use the corresponding grid to calculate the number $p_{(S, D)}$ of paths from $S$ to $D$ as follows:

$$
p_{(S, D)}= \begin{cases}1 & \text { if } \min (\wp)=0 \\ \operatorname{mid}(\wp)+1 & \text { if } \min (\wp)=1 \\ \frac{(\operatorname{mid}(\wp)+1)(\operatorname{mid}(\wp)+2)}{2} & \text { if } \min (\wp)=2\end{cases}
$$

For example, in Figure 5, the number of paths from $S$ to $D$ is determined by two directions $i$ and $j$, as $\left|i_{1}-i_{2}\right|=2$ and $\mid j_{1}-$ $j_{2} \mid=3$ are smaller than $\left|k_{1}-k_{2}\right|=5$. The total number of path alternatives from $S$ to $D$ is 10 . However, one step along the direction of $k$ (it becomes either $k+1$ or $k-1$ ) obviously leads to a longer path. We present two more lemmas on directionrelated properties as follows.

Lemma 4. On a shortest path, the coordinate displacements between $S$ and $D$ along the three directions satisfy

$$
\min (\wp)+\operatorname{mid}(\wp)=\max (\wp)
$$

Proof. The number of hops on a path defined by the correct direction of $\min (\wp)$ and $\operatorname{mid}(\wp)$ is $\min (\wp)+\operatorname{mid}(\wp)$, and the path traverses exactly $\min (\wp)+\operatorname{mid}(\wp)+1$ different points. As each node is intersected by three lines, each from one cluster, one of the three lines must belong to the $\max (\wp)$ cluster. It follows that the path traverses $\min (\wp)+\operatorname{mid}(\wp)+1$ lines in the $\max (\wp)$ cluster. Hence, the displacement of the two line numbers (i.e., $\max (\wp)$ ) traversing $S$ and $D$ is equal to the height of the tree $(\min (\wp)+\operatorname{mid}(\wp)+1)-1$. We have $\max (\wp)=(\min (\wp)+\operatorname{mid}(\wp)+1)-1=\min (\wp)+\operatorname{mid}(\wp)$.

Lemma 5. The distance from source $S$ to destination $D$ is

$$
d(S, D)=\min (\wp)+\operatorname{mid}(\wp) .
$$


The proof of Lemma 5 simply follows the definition of $d(S, D)$ and Lemma 4.

In triangular WMNs, given a source-destination pair $S$ and $D$, the number of shortest paths satisfies the following theorem.

Theorem 6. When $\min (\wp)>0$, the number $p_{(S, D)}$ of paths is determined in a grid of the two correct directions corresponding to $\min (\wp)$ and $\operatorname{mid}(\wp)$ as follows:

$$
p_{(S, D)}=\left(\begin{array}{l}
d(S, D) \\
\min (\wp)
\end{array}\right)
$$

which is the number of $\min (\wp)$ combinations chosen from $d(S, D)$ objects.

Proof. Without loss of generality, let $i$ and $j$ be the correct directions. Note that $i$ is determined by $\min (\wp)$, while $j$ is determined by $\operatorname{mid}(\wp)$. The coordinates of the next receiver node would lead to one hop closer to the destination along the direction $i$ or $j$.

The number $p_{(S, D)}$ of paths is equal to the number of strings of $i$ 's and $j$ 's: $i$ is repeated $\min (\wp)$ times, and $j$ is repeated $\operatorname{mid}(\wp)$ times in a permutation of $\min (\wp)+\operatorname{mid}(\wp)$ elements, that is, $d(S, D)$ in Lemma 5 . The total number of permutations is $d(S, D) !=(\min (\wp)+\operatorname{mid}(\wp)) !$, but with repetitions. Note that the same permutated strings can only be counted once. The number of duplicated permutations is $\min (\wp) !$ and $\operatorname{mid}(\wp)$ ! in the direction of $i$ and $j$, respectively. Then, the total number of different paths is obtained by dividing the total number of permutations by the number of duplications in both directions:

$$
\frac{d(S, D) !}{(\min (\wp) ! \times \operatorname{mid}(\wp) !)}=\left(\begin{array}{c}
d(S, D) \\
\min (\wp)
\end{array}\right)=\left(\begin{array}{c}
d(S, D) \\
\operatorname{mid}(\wp)
\end{array}\right) .
$$

For example, in Figure 5, $\wp=\{2,3,5\}$ with $i$ and $j$ being the correct directions, where $i$ appears $\min (\wp)=2$ times and $j$ appears $\operatorname{mid}(\wp)=3$ times in every permutation. A valid routing path is determined by the number of $i$ 's and $j$ 's as well as their relative positions in the string. For example, $i j j i j$, $i i j j j$, and $i j i j j$ are all valid paths with two $i$ 's and three $j$ 's. The path $p_{1}=S \rightarrow U \rightarrow V \rightarrow W \rightarrow X \rightarrow D$, which is in the set $P(S, D)$ of all shortest paths from $S$ to $D$, can be expressed as a constrained permutation of the two correct directions, that is, $i j j i j$.

Given the node coordinates, data packets are delivered hop by hop along the correct directions, taking the receiver of the current hop as the sender of the next hop, until $\max (\wp)=$ 0 . This can be done recursively and may help avoid the overhead of maintaining a routing table.

4.2. Path Counting with Channel Assignment. For a given path from $S$ to $D$ with a constant number of available orthogonal channels, we need to decide the number of feasible channel assignment schemes for implementing this path by using three channels.

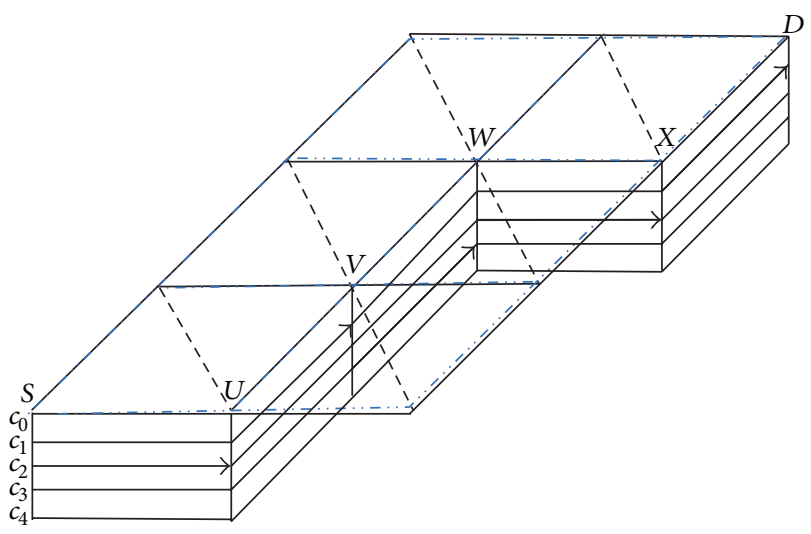

Figure 6: Channel assignment to realize a path from $S$ to $D$.

To illustrate this problem, we show a path with a channel assigned to each hop in Figure 6, which is derived from

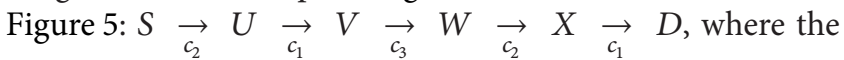
label $c_{i}$ between two neighbor nodes is the channel assigned to the corresponding link.

The directed edges distributed in different layers form a feasible orthogonal channel assignment to a path from $S$ to $D$. A valid path realization allows simultaneous transmission of all the component links on the path. For example, in Figure 6, links $(S, U)_{\mathcal{C}_{2}},(W, X)_{\mathcal{C}_{2}},(U, V)_{\mathcal{C}_{1}},(X, D)_{\mathcal{c}_{1}}$, and $(V, W)_{\mathcal{c}_{3}}$ can coexist at the same time.

Let the correct directions be $i$ and $j$. Given $\emptyset$ orthogonal channels, the first hop has $\left(\begin{array}{l}\infty \\ 1\end{array}\right)$ channel choices, the second hop has $\left(\begin{array}{c}\omega-1 \\ 1\end{array}\right)$ channel choices, and the third hop has $\left(\begin{array}{c}\omega-2 \\ 1\end{array}\right)$ channel choices. Then, the three selected channels can be used repeatedly without interference; that is, the fourth hop selects the same channel as the first hop, and the fifth hop selects the same channel as the second hop and so on. Channel $c_{i}$ can be selected at most $\lceil d(S, D) / 3\rceil$ times.

In realizing a path using three channels at time $t$, the first three hops determine the channel assignment order in every three downstream hops. For example, if the first three hops are arranged in a channel order of $c_{2}, c_{1}$, and $c_{3}$, then the second three hops should be assigned channels in the same order. Otherwise, a realization of the path from $S$ to $D$ would require more channels. For example, with $d(S, D)=4$, if the first three hops use channels in the order of $c_{2}, c_{1}$, and $c_{3}$, the fourth hop cannot use channels $c_{1}$ and $c_{3}$, but only $c_{2}$ or a new channel.

Note that using 3 channels, the channel assignment permutations of the first three hops (i.e., 3!) are all possible schemes for downstream three-hop groups.

Lemma 7. When $\min (\wp) \neq 0$, with $₫$ orthogonal channels, the number of valid channel assignment schemes for a given path from source $S$ to destination D using three channels at the same time is

$$
\lambda=\left(\begin{array}{l}
\varrho \\
3
\end{array}\right) \times 3 !
$$


Based on Theorem 6 and Lemma 7, we have the following theorem.

Theorem 8. When using three channels to realize a path, the number of channel assignments for all shortest paths from $S$ to $D$ is

$$
\left(\begin{array}{c}
d(S, D) \\
\min (\wp)
\end{array}\right) \cdot \lambda
$$

Proof. When $\min (\wp)=0$, there is only one shortest path, and the number of channel assignments for this path is obtained by Lemma 7 .

We focus on a general case where $\min (\wp)>0$. The number of channel assignments can be counted in two independent steps.

The first step is to count all shortest paths from $S$ to $D$ in a plane mesh. This step does not assign channels. For example, Figure 5 shows one such path: $S \rightarrow U \rightarrow V \rightarrow W \rightarrow$ $X \rightarrow D$.

The second step assigns channels to the selected path using three channels without interference. For example, Figure 6 shows the channel assignment for $p_{1}$ from $S$ to $D$ in Figure 5: $S \underset{c_{2}}{\rightarrow} U \underset{c_{1}}{\rightarrow} V \underset{c_{3}}{\rightarrow} W \underset{c_{2}}{\rightarrow} X \underset{c_{1}}{\rightarrow} D$.

Since the above two steps are independent, by the multiplication principle, the total number of path realization schemes satisfies (18). This result could be extended to different numbers of channels assigned to one path.

\section{Conclusion}

We conducted a theoretical exploration on mathematical models and combinatorial characteristics of MIMO WMNs. For a single-channeled mesh, we designed a coordinate scheme and a node identity assignment scheme and derived the interference-free conditions. For multiradio multichannel WMNs, we derived rigorous formulas to count the number of shortest paths from source to destination.

It is of our future interest to find some transformations to generate new link groups from the known ones. Along this direction, we plan to investigate the CPG vertex/edge transitive properties for performance improvement.

\section{Conflict of Interests}

The authors declare that there is no conflict of interests regarding the publication of this paper.

\section{Acknowledgments}

The research is funded in part by Nature Science project of Guangdong Province under Grant no. S2012040010974 and China Scholarship Council no. 201306755013. This research is also partly sponsored by U.S. Department of Energy's Office of Science under Grant no. DE-SC0010641 with University of Memphis.

\section{References}

[1] IEEE Standards Association, "IEEE std. 802.16-2004 for local and metropolitan area networks, part 16: air interface for fixed and mobile wireless access systems," October 2004, http://standards.ieee.org/getieee802/download/802.16-2004.pdf.

[2] I. F. Akyildiz, X. Wang, and W. Wang, "Wireless mesh networks: a survey," Computer Networks, vol. 47, no. 4, pp. 445-487, 2005.

[3] H. Huang, X. Cao, X. Jia, and X. Wang, "Channel assignment using block design in wireless mesh networks," Computer Communications, vol. 32, no. 7-10, pp. 1148-1153, 2009.

[4] Z. Cao, W. Xiao, and L. Peng, "A Mesh $\times$ Chain graph model for MIMO scheduling in IEEE802.16 WMN," in Proceedings of the 2nd IEEE International Conference on Computer Modeling and Simulation (ICCMS '10), vol. 2, pp. 547-551, IEEE, Sanya, China, January 2010.

[5] Z. M. Cao, Y. P. Zhang, Z. L. Shan, and Y. C. Jiang, "A scheme to address routers in MIMO triangular wireless overlay mesh," Applied Mechanics and Materials, vol. 427-429, pp. 2584-2587, 2013.

[6] B. Han, W. Jia, and L. Lin, "Performance evaluation of scheduling in IEEE 802.16 based wireless mesh networks," Computer Communications, vol. 30, no. 4, pp. 782-792, 2007.

[7] F. Jin, A. Arora, J. Hwang, and H.-A. Choi, "Routing and packet scheduling in WiMAX mesh networks," in Proceedings of the 4th International Conference on Broadband Communications, Networks, Systems, (BROADNETS '07), pp. 574-582, Raleigh, NC, USA, September 2007.

[8] Q. Liu, X. Jia, and Y. Zhou, "Topology control for multichannel multi-radio wireless mesh networks using directional antennas," Wireless Networks, vol. 17, no. 1, pp. 41-51, 2011.

[9] A. P. Subramanian, H. Gupta, S. R. Das, and J. Cao, "Minimum interference channel assignment in multiradio wireless mesh networks," IEEE Transactions on Mobile Computing, vol. 7, no. 12, pp. 1459-1473, 2008.

[10] IEEE Standards Association, IEEE Standard for Local and Metropolitan Area Networks-Part 21: Media Independent Handover Services, IEEE Standards Association, 2008, http:// standards.ieee.org/getieee802/download/802.21-2008.pdf.

[11] H. Viswanathan and S. Mukherjee, "Throughput-range tradeoff of wireless mesh backhaul networks," IEEE Journal on Selected Areas in Communications, vol. 24, no. 3, pp. 593-602, 2006.

[12] J. Robinson and E. W. Knightly, "A performance study of deployment factors in wireless mesh networks," in Proceedings of the IEEE 26th IEEE International Conference on Computer Communications (INFOCOM '07), pp. 2054-2062, Anchorage, Alaska, USA, May 2007.

[13] K. Hong and Y. Hua, "Throughput of large wireless networks on square, hexagonal and triangular grids," in Proceedings of the 4th IEEE Workshop on Sensor Array and Multichannel Processing, pp. 461-465, 2006.

[14] M. K. Marina, S. R. Das, and A. P. Subramanian, "A topology control approach for utilizing multiple channels in multi-radio wireless mesh networks," Computer Networks, vol. 54, no. 2, pp. 241-256, 2010.

[15] F. Y. L. Chin, Y. Zhang, and H. Zhu, "A 1-local 13/9-competitive algorithm for multicoloring hexagonal graphs," in Computing and Combinatorics, vol. 4598 of Lecture Notes in Computer Science, pp. 526-536, Springer, Berlin, Germany, 2007.

[16] R. Draves, J. Padhye, and B. Zill, "Routing in multi-radio, multi-hop wireless mesh networks," in Proceedings of the 10th 
ACM Annual International Conference on Mobile Computing and Networking (MobiCom '04), pp. 114-128, October 2004.

[17] H. Tan, T. Lou, Y. Wang, Q.-S. Hua, and F. C. Lau, "Exact algorithms to minimize interference in wireless sensor networks," Theoretical Computer Science, vol. 412, no. 50, pp. 6913-6925, 2011.

[18] Z. Cao and W. Xiao, "An algorithm to generate regular mesh topology for wireless networks," International Journal of Advancements in Computing Technology, vol. 3, no. 3, pp. 123133, 2011.

[19] L. Iannone, R. Khalili, K. Salamatian, and S. Fdida, "Cross-layer routing in wireless mesh networks," in Proceedings of the 1st International Symposium on Wireless Communication Systems (ISWCS '04), pp. 319-323, September 2004.

[20] E. G. Larsson, O. Edfors, F. Tufvesson, and T. L. Marzetta, "Massive MIMO for next generation wireless systems," IEEE Communications Magazine, vol. 52, no. 2, pp. 186-195, 2014.

[21] H. Shetiya and V. Sharma, "Algorithms for routing and centralized scheduling in IEEE 802.16 mesh networks," in Proceedings of the IEEE Wireless Communications and Networking Conference (WCNC '06), vol. 1, pp. 147-152, Las Vegas, Nev, USA, April 2006.

[22] C. Godsil and G. Royle, Algebraic Graph Theory, vol. 207, Springer, New York, NY, USA, 2001.

[23] Z. Xu, W. Qin, Q. Tang, and D. Jiang, "Energy-efficient cognitive access approach to convergence communications," Science China Information Sciences, vol. 57, no. 4, pp. 1-12, 2014.

[24] Z. Cao and L. Peng, "Destination-oriented routing and maximum capacity scheduling algorithms in cayley graph model for wireless mesh network," Journal of Convergence Information Technology, vol. 5, no. 10, pp. 82-90, 2010.

[25] Z. M. Cao and J. C. Tang, "Routing methods and scheduling patterns in MIMO WMN virtual model," Applied Mechanics and Materials, vol. 519-520, pp. 216-221, 2014. 


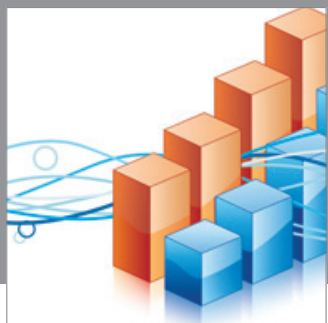

Advances in

Operations Research

mansans

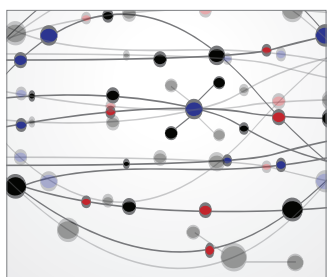

The Scientific World Journal
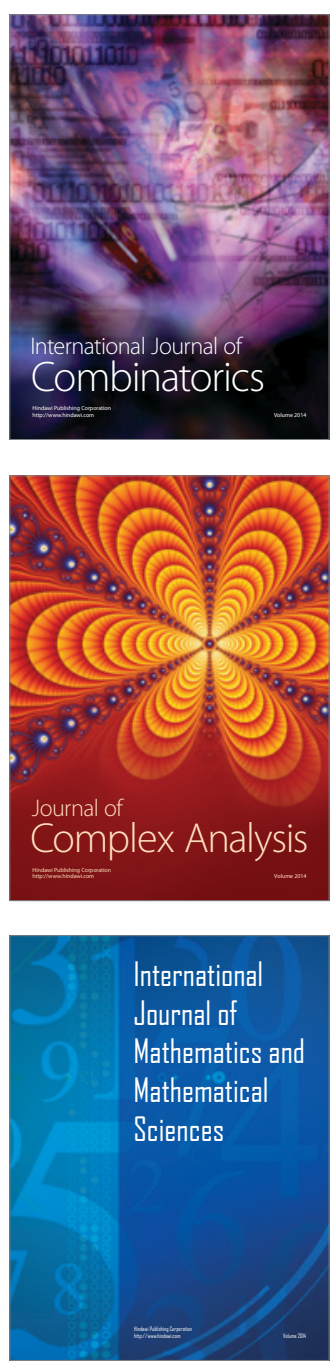
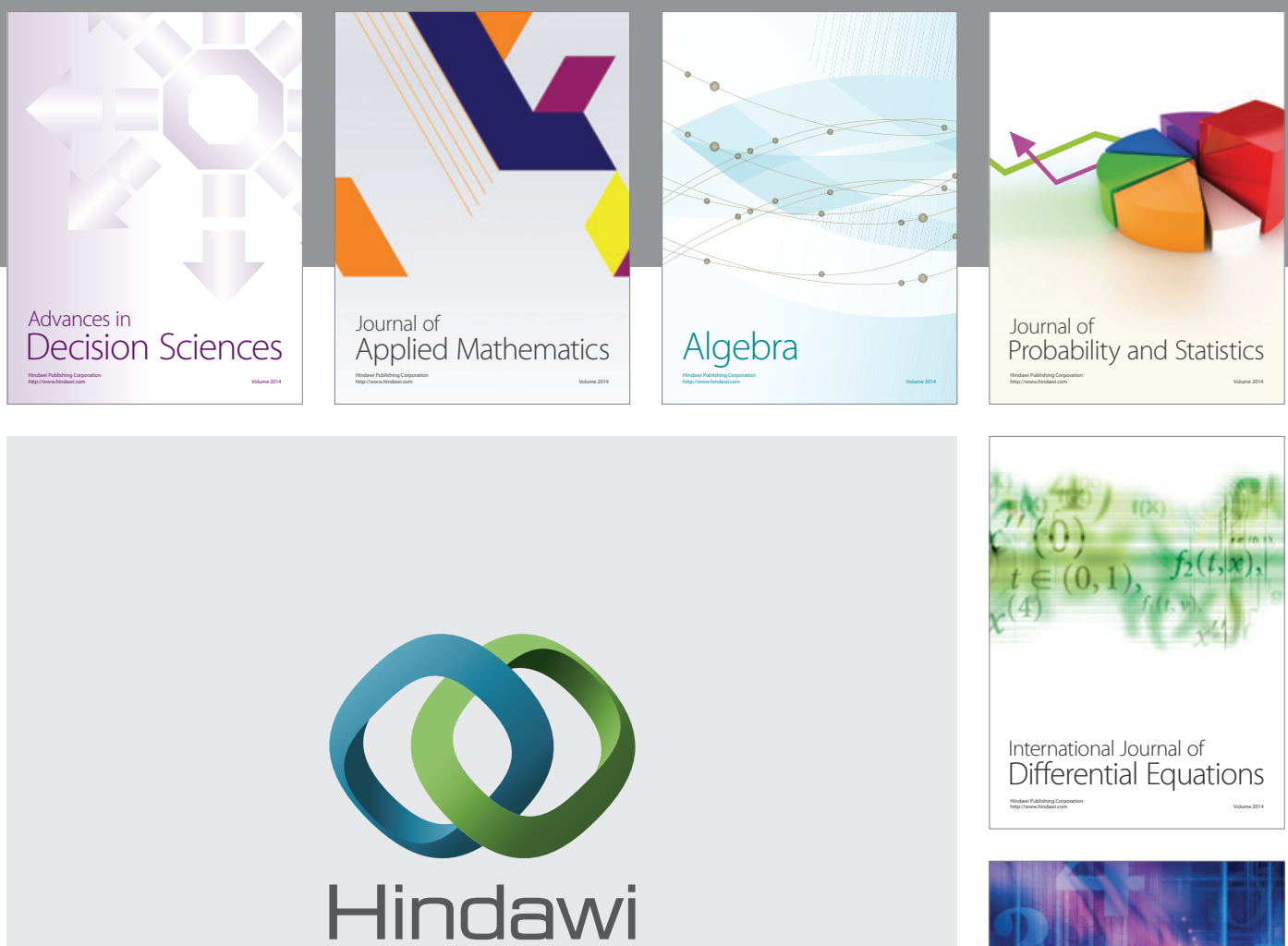

Submit your manuscripts at http://www.hindawi.com
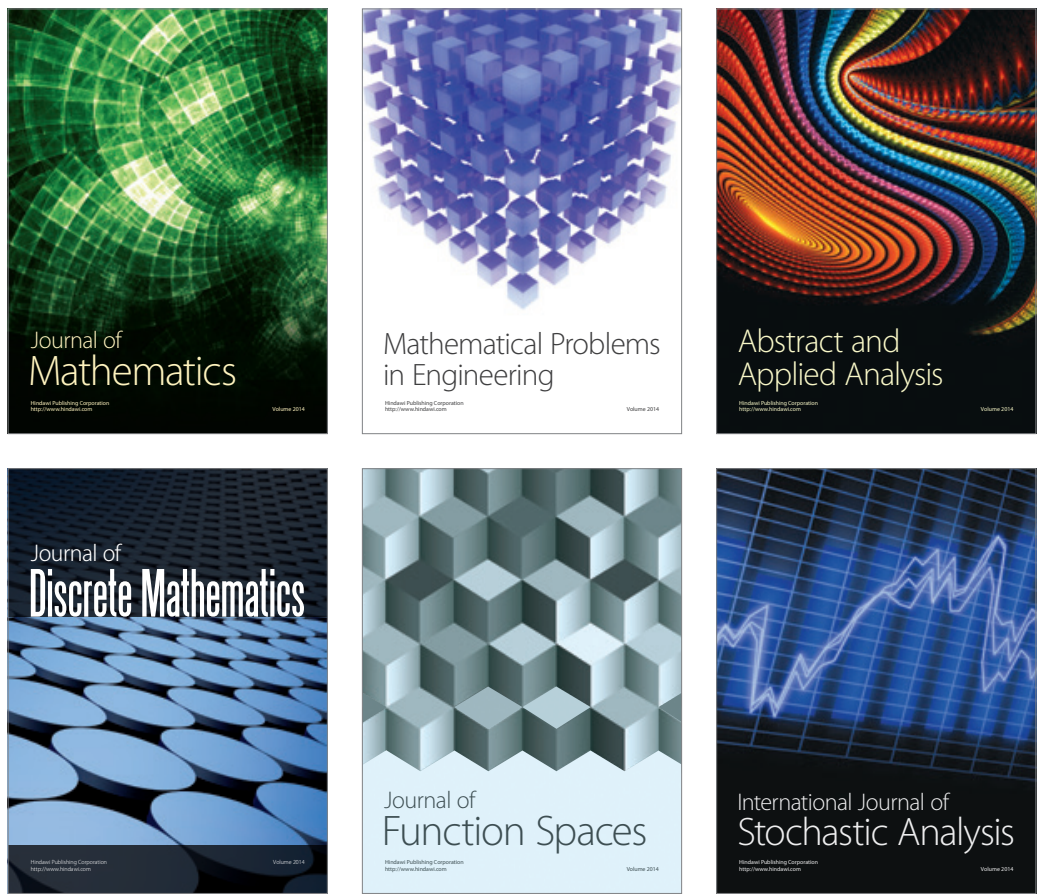

Journal of

Function Spaces

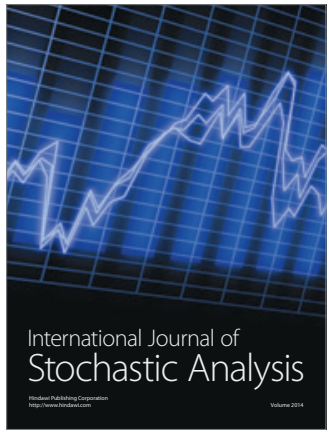

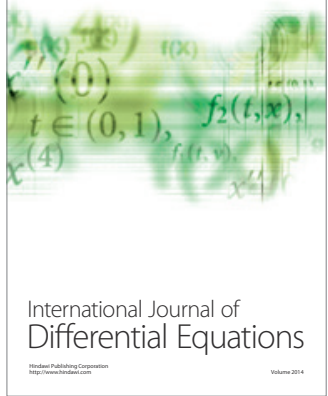
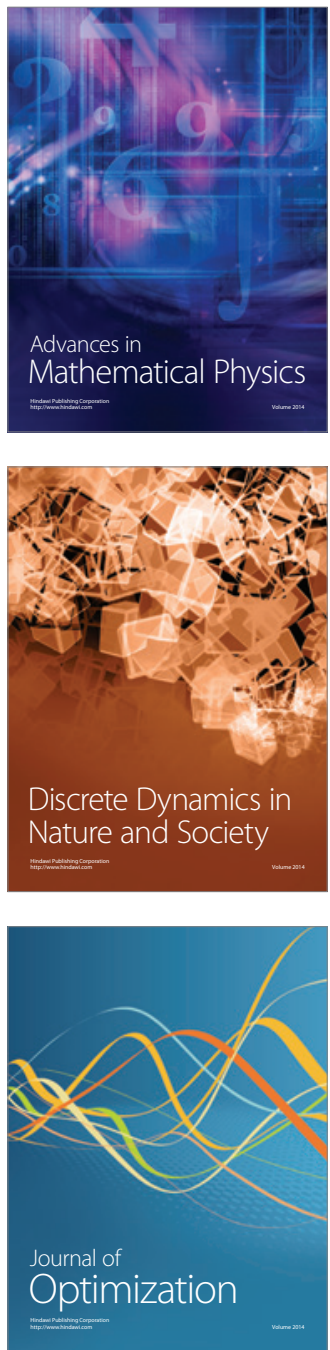